\title{
Editorial
}

\section{Masterclass in airways disease: course report}

Cite as: Spanevello A, Usmani O, Osmonov BR, et al. Masterclass in airways disease: course report. Breathe 2019; 15: e132-e134.

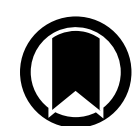

CrossMark
A. Spanevello, O. Usmani, A. Papi, A.A. GarciaNavarro, D. Stolz, G. Brusselle, I. Ojanguren Arranz, M. Cazzola, R. Louis, S. Loukides, S. Desai, A. Nyberg, M. Vendrell

\section{Overview}

It is currently a very exciting time in airway disease. With recent pivotal changes in the global directives in the management for both asthma and COPD, new approved treatments and drugs, and innovative approaches in characterising the diseases, the time was right for assembly 5 (airway diseases, asthma and (OPD) to undertake a masterclass this year in keeping with the new European Respiratory Society (ERS) continuing professional development initiatives from the Educational Council.

Our aim in developing the structure of the masterclass was to provide a "crash course" on the critical updates in asthma and COPD, concentrated over 2 days, and delivered by experts in the field. With participants arriving in Barcelona, Spain, from all continents across the globe and enriched by those engaging online, the scene was set for an exciting 2-day programme (figure 1).

The framework of the masterclass was divided into six key sections: guidelines, assessment and monitoring, practical workshops, disease characterisation, treatments, and clinical cases. The course started with Guy Brusselle and Alberto
Papi highlighting the latest updates in asthma and COPD, respectively, noting the changes in the management of mild asthma and new treatment algorithms in COPD. In the assessment and monitoring session, Inigo Ojanguren Arranz recapped on the importance of lung function in our daily practice, Stelios Loukides reflected on the role of fractional exhaled nitric oxide (among other measures) in his biomarker talk and, in his imaging talk, Sujal Desai reminded us about the key radiological features to help in assessing airway diseases, with particular attention on recognising bronchiectasis.

The practical workshops were, hearteningly, very interactive, with participants particularly eager to learn about airway hyperresponsiveness (from Renaud Louis), exhaled gases and their role in the clinic (from Stelios Loukides), the utility of induced sputum (from Antonio Spanevello) and matching the right inhaler device to the patient (from Omar Usmani). Day 1 ended with a riveting plenary talk delivered by Alvar Agusti entitled "Do airway diseases start in the early life?"

Day 2 started with phenotypes and endotypes of asthma (Renaud Louis) and of COPD (Daiana Stolz), and in the participant discussion these were identified as topics that emerged as most relevant for ongoing future research. In the treatment section, the role of monoclonal biological therapy in severe asthma (Guy Brusselle) and inhaled corticosteroids in COPD (Alberto Papi) were intensely discussed by the participants. We were reminded of the important role of nonpharmacological management in asthma and COPD by Andre Nyberg. The lunchtime plenary

@ERSpublications

Participants attending the recent ERS course on airways disease share their experiences http://bit.ly/2lojSI3 
was entitled "Can bronchiectasis be a negative prognostic factor for airways disease?" and was given by Montserrat Vendrell.

The afternoon clinical cases session was fast, lively and very interactive, with participants encouraged to present their own "complex" cases: in total eight cases were presented and there was great communication among participants in suggesting alterative diagnoses and treatment approaches. In the closing plenary, Mario Cazzola elegantly stepped up and presented " $\alpha_{1}$-antitrypsin deficit: only COPD or asthma as well?"

At the close, participants, chairs and speakers all felt that the masterclass had been a clearly focussed meeting giving an overview of the key developments in airways disease, as well as a forum where experts from around the world were able to meet, understand different clinical healthcare systems and exchange ideas and start collaborations, and, as importantly, have fun while doing so. We very much enjoyed putting together the programme and hope you will be able to join us next time for the assembly 5 (airway diseases, asthma and COPD) masterclass.

\section{Batyr R. Osmonov, Kyrgyzstan, a participant}

The course took place on 13-15 June 2019, again in the historic and beautiful city of Barcelona, Spain.

I just want to note the excellent organisation from the time of registration until the closing of the course, by the ERS education team. During the course, it was very comfortable, I would even say a family atmosphere. The multiple-choice questions presented at the end of each presentation gave the opportunity for the audience to interact with the faculty and even discuss cases in further details.

One very important moment during this event was the opportunity to network and build new relationships. Having the chance to meet with colleagues from all over the world to share best practices was very valuable.

It is impossible to underestimate the benefits of this masterclass for practitioners and young scientists. Everyone could find what was necessary and interesting for him: from general principles, approaches to the treatment of major obstructive diseases, to the subtleties of the approach to managing patients with rare obstructive diseases, rehabilitation of patients with severe concomitant pathologies, etc. There was an opportunity to learn new trends in the field of diagnostics, which is just as important for the practical work of a doctor.

This is not to mention the great experience that I received as a young scientist. The case-based sessions really helped in understanding the different perspectives and angles from which we can look at a case, therefore giving us practical advice and many recommendations.

As for the subject matter of the masterclass, it is impossible to divide the topics into important

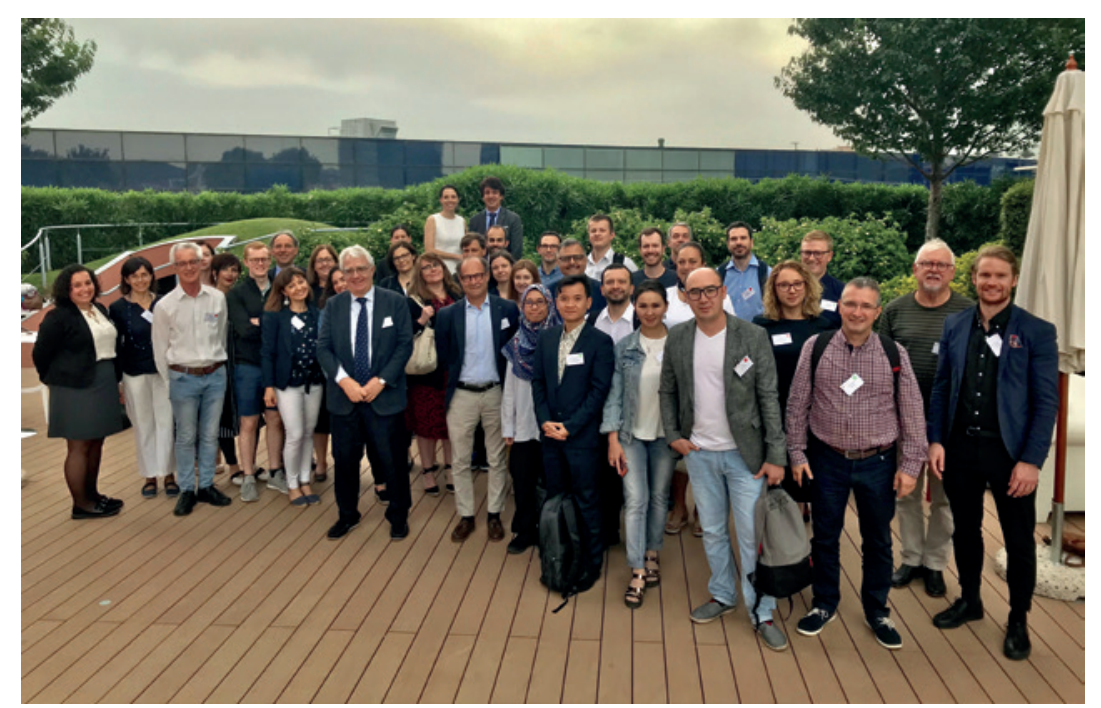

Figure 1 Faculty and participants in Barcelona for the masterclass in airways disease.

and/or less important, since each topic, each presentation, was invaluable and important for any doctor. We can start with the report of Guy Brusselle and Alberto Papi on new updates of clinical recommendations on bronchial asthma and COPD. A lot of publications on these pathologies are often contradicting one another and it is sometimes difficult to determine which strategy to undertake. These presentations were very clear and showed us how to act and help our patients.

Subsequent to that, we talked about the importance of spirometry, biomarkers and diagnostic imaging in obstructive diseases. I also want to flag the great debate we had on the themes of spirometry and $\mathrm{X}$-ray diagnostics. Very often, many pulmonologists encounter mixed spirometry indicators, both obstructive and restrictive, and the question arises as to what to treat first and what problem prevails in the patient and I think all participants found answers to their questions in this session.

There was an interesting opinion that it is necessary to conduct spirometry at an early stage, starting in schools, for the early detection and prevention of obstructive diseases. Especially since with each new generation we get a deformed genotype and a predisposition to new and already known respiratory diseases.

The session on inhalation technology was very interesting and interactive. A video was shown about the inadequacy of the inhalation technique and, as well, all the participants conducted training among themselves: how to show the patient the correct inhalation technique. Personally, there were many new facts about the correctness of performance and effectiveness of different types of inhalers.

From the first day after I returned home, I started using my new knowledge and ideas, and every day I apply the experience gained in my practice. I also conducted a small masterclass among my colleagues so that I could share the knowledge I received. 
I express my greatest gratitude to the organisers of this masterclass and to the ERS for the opportunity to participate in such a good event. I would like to ask them to carry out further educational schools and masterclasses on various topics of respiratory medicine and to attract doctors and young scientists from developing countries with limited opportunities to obtain best practices and the latest updates.

\section{Kostiantyn Dmytriiev, Ukraine, a participant}

A friendly atmosphere was present throughout the course, which provided a nice context to share best practices and build new relationships.

Most of the topics covered during the course were of the greatest relevance and importance for daily clinical practice, while others were setting new horizons for the future of scientific research. Each topic gave new insight and broadened vision on both COPD and asthma. More specifically, I would like to mention the last lecture of the first day: "Do airway diseases start in the early life?" given by Alvar Agusti. In my opinion, it was eye-opening. Alvar Agusti went back to the roots of the lung function development and decline throughout life, which are relevant for clinical practice and for future research as well.

Each topic at the course covered a small part of a bigger picture and each topic shaped this picture of asthma or COPD management, going back from the underlying pathological changes to their phenotype manifestations, diagnostic approaches and best treatments available. Both lectures on clinical guidelines provided information not only on guidelines themselves, but also on the statements that can be the subject of future research. A block of lectures on assessment and monitoring airways disease, as well as masterclasses, provided tips for better management in these diseases. Lectures on phenotyping and endotyping of COPD and asthma provided useful information for the assessment and differentiation of manifestations of these diseases, which is very important. Updates on different types of treatment are important and we discussed the opportunities to implement some of them in everyday practice.

The course outlined the importance of medical history, especially in early life, and provided an understanding of disease progression. Diagnostic work-up and treatment plans can be improved with the new information received, especially regarding the importance of inhaler use technique in the management of airway diseases.

\section{Kseniia Suska, Ukraine, a participant}

The warmest and friendliest atmosphere of all the events I have been fortunate to participate in. There were a lot of respectful experts in the field of airway diseases who shared their scientific and clinical experience with the participants of the masterclass. Having all these participants and faculty from all over the world allowed us to share our best practices and build new relationships.

During the course, topics such as the management of asthma and COPD patients, as well as lung imaging, were very interesting and useful as we face these questions every day in our clinical practice. The case-based session turned out to be very exciting as it allowed us to discuss interesting and difficult cases from daily practice. The interaction in this session gave us the opportunity to receive good advice from leading experts. The practical workshops on induced sputum, inhalation techniques, exhaled gases, airway hyperresponsiveness and its practical clinical application have been incredibly useful, and I plan to apply this knowledge in my clinical practice.

During this course I realised that the clinical condition of the patients is the main biomarker, which should be decisive in choosing the strategy for managing patients with airway diseases.

I would like to thank the ERS and the organising committee of the masterclass for the opportunity to participate in such an informative scientific event.

\title{
Affiliations
}

\author{
Antonio Spanevello', Omar Usmani², Batyr R. Osmonov³, Kostiantyn Dmytriiev ${ }^{4}$, Kseniia Suska \\ ${ }^{1}$ University of Insubria, Varese, Italy. ${ }^{2}$ Imperial College London, London, UK. ${ }^{3}$ Dept of Internal Medicine, \\ International Medical University of Kyrgyzstan, Medical Center of the Kyrgyz State Medical Academy, named after \\ I.K. Ahunbaev, Bishkek, Kyrgyzstan. ${ }^{4}$ National Pirogov Memorial Medical University, Vinnytsia, Ukraine. ${ }^{5}$ State \\ Institution Dnipropetrovsk Medical Academy of the Ministry of Health of Ukraine, Dept of Occupational Diseases \\ and Clinical Immunology, Dnipro, Ukraine.
}

\section{Conflict of interest}

A. Spanevello has nothing to disclose. O. Usmani reports grants and personal fees from AstraZeneca, Boehringer Ingelheim and Chiesi, personal fees from Aerocrine, Napp, Mundipharma, Sandoz, Takeda, Zentiva, Cipla and Pearl Therapeutics, and grants from GlaxoSmithKline, Prosonix and Edmond Pharma, outside the submitted work. B.R. Osmonov has nothing to disclose. K. Dmytriiev has nothing to disclose. K. Suska reports grants from ERS (bursary to cover the course registration fee), during the conduct of the study. 